\title{
Gender differences in anxiety and depression among the caregivers of patients with dementia
}

\author{
Rohit Verma $^{1^{\star}}$, Kuljeet Singh Anand ${ }^{2}$ \\ ${ }^{1}$ Department of Psychiatry, Post Graduate Institute of Medical Education and Research, Dr. Ram Manohar Lohia Hospital, New \\ Delhi, India; *Corresponding Author: rohit.aiims@gmail.com \\ ${ }^{2}$ Department of Neurology, Post Graduate Institute of Medical Education and Research, Dr. Ram Manohar Lohia Hospital, New \\ Delhi, India
}

Received 24 October 2012; revised 28 November 2012; accepted 6 December 2012

\section{ABSTRACT}

Background: Providing care to dementia patients itself poses risk of distress in bio-psychosocial paradigm. It is important to assess the mental health status and attend to the needs of caregivers. The present investigation focuses on gender differences related to anxiety and depression in caregivers of dementia patients. Methods: This cross-sectional study was carried out in an out-patient setting in the Dementia clinic of a tertiary care hospital. Sixty caregivers of consescutive dementia patients receiving outpatient care were assessed using the Hospital Anxiety and Depression Scale (HADS). Descriptive analysis was done to find in between group differences for the male and female caregivers and Pearson's correlation coefficient was calculated to find the association between the number of family members with chronic illness and the scores on HADS. Results: Male and female caregivers differed significantly on the HADS-Total, Anxiety and Depression subscale scores with female care givers having significantly higher scores. There was a significant correlation between the number of family members with chronic illness and HADS-Anxiety subscale scores in female caregivers. Conclusion: It is crucial to screen caregivers, especially females, of patients with dementia for presence of anxiety and depression. This approach will help timely identification and proper management of these individuals. It is important to promote measures to soften the impact that the patient has on the caregiver, and that, at the same time, improves the quality of life of the patient.

Keywords: Caregiver; Anxiety; Depression; Dementia; Gender

\section{INTRODUCTION}

With the developing world witnessing a demographic transition, dementia disorders, which already are a major cause of burden of disease amongst the elderly, are likely to account for an even greater proportion of this burden in the future [1]. The 10/66 dementia research group reported the prevalence of dementia in India and South Asia as $1.9 \%$ in those $\geq 60$ years with an annual incidence of 4.3/1000 and it estimated to reach 3.6 million mark by 2020 [2]. The rate of increase was estimated to be 3 - 4 times higher in developing countries than in developed countries [2].

Initiating as a cognitive decline, dementia consequently paves way to a marked change in the neuropsychological parameters further progressing as neuropsychological and behavioral disturbances [3]. With time, it manifests increasing difficulty in carrying out activities of daily living, making the individual more and more dependent on the caregivers for support. Family members, who often provide the first line of care, often need to sacrifice their own health and well-being while providing extensive care to the patients. The progressive mental and physical deterioration increases the need for constant care. In the worst scenario, taking care of the patient becomes a full-time occupation for the caregiver. The resulting constant stress in caregivers puts them more at risk of compromising their own physical and psychosocial well-being. Not only do the care givers impact their own health and well being, their ill health impacts the care of the patients as well.

There is evidence of considerable physical and psychological morbidity like hypertension, anxiety and depression in caregivers of dementia patients which further reduces their work capacity with higher strain, increased burden and impaired quality of life [4-8]. Indian data also suggests high correlation between behavioural problems in patients with dementia and psychological distress and poor quality of life in their caregivers [9]. Research has found spouses of patients with dementia to be 
at higher risk of social isolation, emotional burden and a reduction in quality of life [10].

There is an urgent need to focus attention, in particular, on the detrimental effects that the caring for dementia patients has on the caregiver. There is currently a dearth of data in this context from developing countries. The current study aims at assessment of the gender differences related to depression and anxiety levels among the caregivers of patients with dementia being treated in an out-patient setting of a developing country.

\section{METHODS}

The study was carried out in an outpatient setting in the Dementia clinic, Department of Neurology, Post Graduate Institute of Medical Education and Research, Dr. Ram Manohar Lohia Hospital, New Delhi, India. Sixty consecutive caregivers of dementia patients receiving outpatient care were included in the study. All caregivers willing to participate in the study and giving informed consent were included in the study. To be eligible for participation in the study the family member present with the patient had to be the primary caregiver. A person was considered a primary caregiver (spouse or relative) when he/she was knowledgeable about the person with dementia and spent a minimum of 4 hours a week in direct contact (face to face) with them. Only one person was considered the nominated caregiver. Socio-demographic data of the caregivers were collected using a semi-structured proforma.

The anxiety and depression levels of these individuals were assessed using the Hospital Anxiety and Depression Scale (HADS). HADS is a self-report questionnaire commonly used to assess levels of anxiety and depression [11]. The HADS comprises statements which the patient rates based on their experience over the past week. The 14 statements are relevant to generalized anxiety (7 statements) or "depression" (7 statements). Each question has 4 possible responses. Responses are scored on a scale from 3 to 0 . The two subscales, anxiety (HADS-A) and depression (HADS-D), have been found to be independent measures. In its current form the HADS is now divided into four ranges: normal $(0-7)$, mild $(8-10)$, moderate (11 - 15) and severe (16 - 21).

The HADS questionnaire has been translated into many languages, and for many of these translations validation studies confirm the internationally applicable nature of this Questionnaire [12]. The HADS-A has been found to have an optimal cut off of 8 (sensitivity 0.89 , specificity 0.75 ) in relation to generalized anxiety and HADS-D has been found to have optimal cut off of 8 (sensitivity 0.80 and specificity 0.88 ) in relation to major depressive episode in general practice patients [13]. HADS has previously been used for caregivers of people with dementia [14]. It does not include somatic items, which can be misleading in older people.

The data were imputed and analyzed using SPSS ver17.0. Descriptive analysis was done for the socio-demographic profile and independent sample t test was carried out to find in between group differences for the male and female caregivers. The level of statistical significance was kept at $p<0.05$. Pearson's correlation coefficient was calculated to find the association between the number of family members with chronic illness and the scores on HADS.

\section{RESULTS}

We recruited the primary caregiver for each of 60 consecutive out-patients receiving care in the dementia clinic of neurology department of a tertiary care hospital.

The sample included 36 male (60\%) and 24 female $(40 \%)$ caregivers. The characteristics of caregivers (study population) are outlined in Table 1 . The mean $( \pm \mathrm{SD})$ age of male and female caregivers was $46.11( \pm 16.19)$ years and $58.83( \pm 20.8)$ years respectively with no significant difference amongst the groups.

All of the subjects were literate, from urban background, and belonged to middle socio-economic status. The entire male and half of female caregivers were the primary earning members of the family as well. In $66 \%$ of male and $75 \%$ of female caregivers, there were other family members with chronic illness for whom too they were the primary caregivers. There were no significant

Table 1. Socio-demographic profile of the study population.

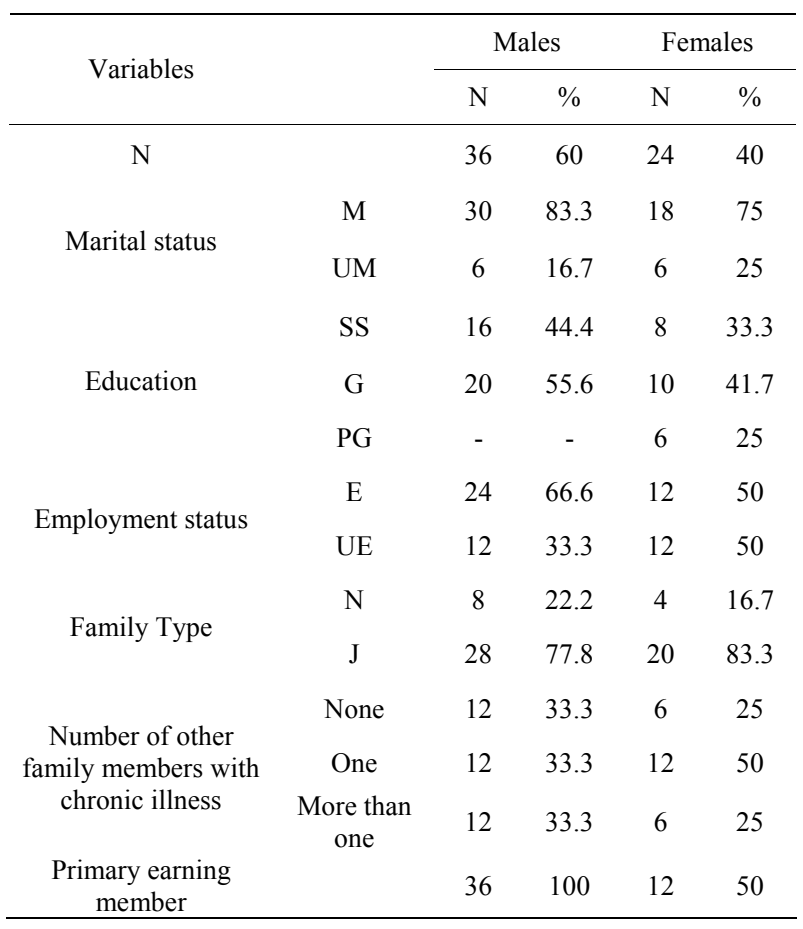

M: Married; UM: Unmarried; SS: Senior Secondary; G: Graduation; PG: Post Graduation; E: Employed; UE: Unemployed; N: Nuclear; J: Joint. 
differences amongst the male and female groups on any socio-demographic parameter.

The mean duration of dementia care giving in male and female groups was not significantly different being $5.78( \pm 6.73)$ years and $5.58( \pm 3.08)$ years respectively. Male and female caregivers differed significantly on the total HADS score as well as the Anxiety and Depression subscale scores of HADS with the female care givers having significantly higher scores. Table 2 provides the findings of the independent sample $t$ test. Additionally there was a significant correlation between the number of family members with chronic illness and HADS-Anxiety subscale scores in female caregivers (Pearson's correlation coefficient $0.37 ; \mathrm{p}<0.05$ ). However there was no correlation seen with total HADS score and HADS Depression subscale score in both groups.

\section{DISCUSSION}

The current study found that more males than females served as primary caregivers to dementia patients. Studies from developed and developing countries have reported of women serving as caregivers far more frequently than men $[15,16]$. Indian studies also report of similar findings as western studies [9]. But Indian data have been from rural areas, which may have a more female care oriented nature with the males working. Our study was from an urban area, where the changing demographic pattern of increasing participation of females as earning member of family may have partly played a role in shifting the care giving dynamics to male members of the society. As was observed in our study, about $50 \%$ of female caregivers were the primary earning members of the family.

With similar duration of care giving in both groups and even though males outnumbered female caregivers, the levels of both anxiety and depression were observed to be significantly higher among female caregivers as compared to the male caregivers in our study.

The results of studies comparing outcomes for male versus female caregivers have been variable. Where some

Table 2. Independent sample t-test for in between group differences between male and female caregivers on HADS scale scores.

\begin{tabular}{cccccc}
\hline & & & & \multicolumn{2}{c}{$95 \%$ Confidence Interval } \\
\cline { 5 - 6 } Scale & $\mathrm{T}$ & P value & MD & Lower & Upper \\
\hline HADS total score & 2.29 & $0.029^{\mathrm{a}}$ & -7.14 & -13.5 & -0.78 \\
$\begin{array}{c}\text { HADS anxiety } \\
\text { score }\end{array}$ & 2.32 & $0.027^{\mathrm{a}}$ & -3.28 & -6.16 & -0.39 \\
$\begin{array}{c}\text { HADS depression } \\
\text { score }\end{array}$ & 2.06 & 0.048 & -3.86 & -7.69 & -0.03 \\
\hline
\end{tabular}

MD: Mean Difference; HADS: Hamilton Anxiety Depression Scale; ${ }^{\mathrm{a}} \mathrm{P}$ value $<0.05$. have found the effects to be worse for women $[17,18]$ others have found the effects to be either worse for men or similar for the two [19,20]. A study on 200 caregivers and their patients reported that the caregivers were usually female, who care for the sick at home and of these, $55 \%$ had problems with the patient's family and/or their own family, and 57\% at work [16]. The increase in the degree of anxiety and depression was reported directly proportional to the severity of the illness, affecting the patient [16].

A qualitative study by the Indian 10/66 Dementia Research Network reported that the majority of caregivers were young women and the principal sources of caregiver strain were behavioural problems associated with the dementia syndrome and incontinence leading to significant deterioration in their mental health [9]. Strain was exacerbated by the lack of supportive response by local health services, and by lack of support and, sometimes, criticism from other family members.

In another study, stress, anxiety and depression were positively correlated with caring hours, sleep problems in caregivers and behavioural disturbances of the patients; and were negatively correlated with free time and time spent out of the home [21]. Studies report that about $61 \%$ of caregivers have their health affected and nearly all have medical problems, including arthritis, high blood pressure, anxiety and depression [22]. They were afraid of the future, without enough time for themselves and their own social life, frequently or always burdened by caring and stressed, and felt they had lost control of their lives [22]. In yet another study, 32\% caregivers of dementia patients were found depressed and the patient predictors of caregiver depression included younger age, white and Hispanic ethnicity, less than a high school education, Activities of Daily Living dependence, and behavioral disturbance, particularly angry or aggressive behavior [17]. Independent caregiver predictors of depression included low income, relationship to the patient, hours spent caregiving and functional dependence [17].

It was also observed that females significantly suffered more anxiety with increasing number of family members having chronic illness, while male caregivers did not. It may be due to a reduced coping ability in female caregivers as compared to males generated by increasing duties, demands and responsibilities as a result of care giving. Increased rates of depression and anxiety have been observed in female caregivers of mentally ill patients when compared with the female non-caregivers in the community [23].

Meta-analysis of studies conducted in various countries has shown that women are roughly twice as likely as men to experience depression [24]. The reason for this sex difference could be attributed to various biological, 
environmental, and psychological factors such as: the effects of hormones like estrogen on stress, dependence on relationships, female victimization, higher societal expectations of gender role adherence, lesser aggressiveness, faulty attribution styles and ruminative coping [24]. Viewed from a life course perspective, gender influences life experiences, psychological development and functioning and, therefore, can result in different psychologycal developmental patterns [25]. Providing care to dementia patients may pose as an extra burden in female population of the society, thereby increasing preponderance of minor mental health illnesses.

Psychological interventions with dementia patients and their caregivers in India are at the initial phase with most interventions being carried out in institutionalized patients [26]. But, most Indian families cannot afford institutional care, which in any case is, unavailable in most parts of the developing world, and so people with dementia are cared for at home, by their families, where spouses are the primary caregiver, making home-based programs a more beneficial solution to cater this population. Considering caregiver distress in dementia and their role in patient care, an urgent attention to this aspect is needed. Attention is also needed on females who may be suffering more than their male counterparts in providing care to dementia patients.

Though the small sample size of the study limits its extrapolation to the population at large, it does transmit the presence of caregiver anxiety and depressive illnesses in this population, more so among female gender. Since the duration of illness was neither too long nor too significantly different amongst the groups, the study could not predict the actual impact of a long standing dementia.

\section{CONCLUSIONS}

Dementia involves not only the patient, but also affects the whole family. Moreover, treatment complexity; commitment constancy of the caregiver; inadequacy of the public service; direct and indirect costs of care; and ensuing effects upon emotional and interpersonal relations also play a major role in influencing the psychological, social and physical wellbeing of the caregiver.

The present research highlights and confirms the presence of anxiety and depressive illness in caregivers of dementia subjects, in those families that have to take care of patients who are not hospitalized. It is vital to screen the caregivers, especially female caregiver, of the patients with dementia for presence of anxiety and depression. This approach will help timely identification and proper management of these individuals. The planning and putting into practice of support interventions, guidance and aid to families could be a valid solution to the caregiver's distress.

\section{ACKNOWLEDGEMENTS}

R.V. thanks Mr. Rajat Sahni and Mrs. Jolly Sahni for the valuable support needed.

\section{REFERENCES}

[1] Prince, M., Graham, N., Brodaty, H., et al. (2004) Alzheimer disease international's 10/66 dementia research group-One model for action research in developing countries. International Journal of Geriatric Psychiatry, 19, 178-181. doi:10.1002/gps.1059

[2] Ferri, C.P., Prince, M., Brayne, C., et al. (2005) Global prevalance of dementia: A Delphi consensus study. Lancet, 366, 2112-2117. doi:10.1016/S0140-6736(05)67889-0

[3] Finkel, S.I. (2001) Behavioural and psychological symptoms of dementia: A current fours for clinicians, researchers and caregivers. The Journal of Clinical Psychiatry, 62, 3-6.

[4] Dunkin, J.J. and Anderson-Hanley, C. (1998) Dementia caregiver burden: A review of the literature and guidelines for assessment and intervention. Neurology, 51, 5360. doi:10.1212/WNL.51.1_Suppl_1.S53

[5] Aguglia, E., Onor, M.L., Trevisiol, M., et al. (2004) Stress in the caregivers of Alzheimer's patients: An experimental investigation in Italy. American Journal of Alzheimer's Disease and Other Dementias, 9, 248-252. doi: $10.1177 / 153331750401900403$

[6] Torti Jr., F.M., Gwyther, L.P., Reed, S.D., et al. (2004) A multinational review of recent trends and reports in dementia caregiver burden. Alzheimer Disease and Associated Disorders, 18, 99-109. doi:10.1097/01.wad.0000126902.37908.b2

[7] Cooper, C., Balamurali, T.B. and Livingston, G. (2007) A systematic review of the prevalence and covariates of anxiety in caregivers of people with dementia. International Psychogeriatrics, 19, 175-195. doi:10.1017/S1041610206004297

[8] Fortinsky, R.H., Tennen, H., Frank, N. and Affleck, G. (2007) Health and psychological consequences of caregiving. In: Aldwin, C.M., Park, C.L. and Spiro, R., Eds., Handbook of Health Psychology and Aging, Guilford Press, New York, 227-249.

[9] Shaji, K.S., Smitha, K., Lal, K.P. and Prince, M.J. (2003) Caregivers of people with Alzheimer's disease: A qualitative study from the Indian 10/66 Dementia Research Network. International Journal of Geriatric Psychiatry, 18, 1-6. doi:10.1002/gps.649

[10] Thommessen, B., Aarsland, D., Braekhus, A., et al. (2002) The psychosocial burden on spouses of the elderly with stroke, dementia and Parkinson's disease. International Journal of Geriatric Psychiatry, 17, 78-84. doi:10.1002/gps.524

[11] Zigmond, A.S. and Snaith, R.P. (1983) The hospital anxiety and depression scale. Acta Psychiatrica Scandinavica, 67, 361-370. doi:10.1111/j.1600-0447.1983.tb09716.x 
[12] Herrmann, C. (1997) International experiences with the hospital anxiety and depression scale-A review of validation data and clinical results. Journal of Psychosomatic Research, 42, 17-41. doi:10.1016/S0022-3999(96)00216-4

[13] Olsson, I., Mykletun, A. and Dahl, A.A. (2005) The hospital anxiety and depression rating scale: A cross-sectional study of psychometrics and case finding abilities in general practice. BioMed Central Psychiatry, 5, 46.

[14] Bjelland, I., Dahl, A.A., Haug, T.T. and Neckelmann, D. (2002) The validity of the hospital anxiety and depression scale: An updated literature review. Journal of Psychosomatic Research, 52, 69-77. doi:10.1016/S0022-3999(01)00296-3

[15] Campbell, L.D. and Martin-Matthews, A. (2003) The gendered nature of men's filial care. The Journals of Gerontology. Series B, Psychological Sciences and Social Sciences, 58, 350-358. doi:10.1093/geronb/58.6.S350

[16] Ferrara, M., Langiano, E., Di Brango, T., et al. (2008) Prevalence of stress, anxiety and depression in with Alzheimer caregivers. Health and Quality of Life Outcomes, 6, 93. doi:10.1186/1477-7525-6-93

[17] Covinsky, K.E., Eng, C., Lui, L.Y., et al. (2001) Reduced employment in caregivers of frail elders: Impact of ethnicity, patient clinical characteristics, and caregiver characteristics. The Journals of Gerontology. Series A, Biological Sciences and Medical Sciences, 56, 707-713. doi:10.1093/gerona/56.11.M707

[18] Cuijpers, P. (2005) Depressive disorders in caregivers of dementia patients: A systematic review. Aging and Mental Health, 9, 325-330. doi:10.1080/13607860500090078

[19] Schulz, R. and Williamson, G.M. (1991) A 2-year longi- tudinal study of depression among Alzheimer's caregivers. Psychology and Aging, 6, 569-578. doi:10.1037/0882-7974.6.4.569

[20] Savla, J., Almeida, O.M., Davey, A. and Zarit, S.H. (2008) Routine assistance to parents: Effects on daily mood and other stressors. The Journals of Gerontology. Series B, Psychological Sciences and Social Sciences, 63, 154-161. doi:10.1093/geronb/63.3.S154

[21] Vellone, E., Piras, G. and Sansoni, J. (2002) Stress, anxiety, and depression among caregivers of patients with Alzheimer's disease. Annali Di Igiene, 14, 223-232.

[22] Bridges-Webb, C., Giles, B., Speechly, C., et al. (2006) Patients with dementia and their carers in general practice. Australian Family Physician, 35, 923-924.

[23] Cherkil, S. (2010) Coping styles, stress tolerance, and wellbeing and their correlations in the women spouses of the mentally ill. Indian Journal of Psychological Medicine, 32, 99-103.

[24] Nolen-Hoeksema, S. and Girgus, J.S. (1994) The emergence of gender differences in depression during adolescence. Psychological Bulletin, 115, 424-443. doi:10.1037/0033-2909.115.3.424

[25] Hammen, C. (2003) Mood disorders. In: Stricker, G. and Widiger, T., Eds., Handbook of Psychology, Clinical Psychology (Volume 8), John Wiley and Sons, Inc., New York, 93-118.

[26] Dias, A., Dewey, M.E., D’Souza, J., et al. (2008) The effectiveness of a home care program for supporting caregivers of persons with dementia in developing countries: A randomised controlled trial from Goa, India. PLoS One, 4, e2333. doi:10.1371/journal.pone.0002333 